\title{
Recognition of boards using wood fingerprints based on a fusion of feature detection methods
}

\author{
Tobias Pahlberg, ${ }^{\mathrm{a}, *}$, Olle Hagman ${ }^{\mathrm{a}}$, Matthew Thurley ${ }^{\mathrm{b}}$ \\ ${ }^{a}$ Luleå University of Technology, Campus Skellefteå, \\ Forskargatan 1, SE-931 87 Skellefteå, Sweden \\ ${ }^{b}$ Luleå University of Technology, \\ SE-971 87 Luleå, Sweden
}

\begin{abstract}
This paper investigates the possibility to automatically match and recognize individual Scots pine (Pinus sylvestris L.) boards using a fusion of two feature detection methods. The first method denoted Block matching method, detects corners and matches square regions around these corners using a normalized Sum of Squared Differences (SSD) measure. The second method denoted the SURF (Speeded-Up Robust Features) matching method, matches SURF features between images (Bay et al., 2008). The fusion of the two feature detection methods improved the recognition rate of wooden floorboards substantially compared to the individual methods. Perfect matching accuracy was obtained for board pieces with more than 20 knots using high quality images. More than $90 \%$ matching accuracy was achieved for board pieces with more than 10 knots, using both high- and low quality images.
\end{abstract}

Keywords: Wood fingerprint, Traceability, Feature detection, Biometrics, Hol-i-Wood Patching Robot

\section{Introduction}

The motivation for this work is: (i) to find out if it is possible for a machine vision system to correctly reidentify wooden boards using only their biometric "fingerprint", and (ii) to find out exactly how small wood pieces can be recognized using a fusion of so called feature detection methods.

This is a continuation of the work done by Pahlberg and Hagman (2012) and is likewise a part of the Hol-iWood Patching Robot project. The project outcome will consist of several different holonic modules. Holonic, means that something is simultaneously a part of something whole, but can still work by itself, e.g., like a human cell. The wood fingerprint recognition system is such a part.

This particular application is supposed to run in realtime, meaning that the processing unit is only allowed a few hundredths of a second to correctly identify the wood piece when it arrives at one of the patching robots. The search space will consist of all the scanned wood

\footnotetext{
${ }^{*}$ Corresponding author. Address: Luleå University of Technology, Campus Skellefteå, Forskargatan 1, SE-931 87 Skellefteå, Sweden. Telephone: +46 (0)910-585336

Email address: tobias .pahlberg@ltu.se (Tobias Pahlberg)
}

products that are, so to say, in the flow and on their way to being patched. This is therefore a so called closedset identification task, where the sought "individual" is known to be in the database.

\subsection{Traceability in the wood chain}

The wood industry has been investigating solutions to a few traceability problems in the past. Efforts have been put into investigating the possibility of tracking trees between harvesting and sawmills using RFID tags (Björk et al., 2011; Häkli et al., 2013), tracking logs between the log sorting station and the saw intake (Chiorescu and Grönlund, 2004) and identifying which boards originate from which logs (Flodin et al., 2008). Attempts to recognize boards using board end images have also been carried out (Põlder et al., 2012). Other invasive technologies like barcode stickers and sprayed on paint have also been investigated (Dykstra et al., 2002).

One big gain with traceability of wood products would be the possibility for direct error feedback (Grönlund, 2008). If something is wrong with the end product, if it has the wrong moisture content, if the yield is low, there are possibilities to trace back through the chain and easier localize the origin of the problem. 
Traceability would make it possible to do on-line and instantaneous calibration of machines. Today, time consuming and expensive test sawings are needed to calibrate machines and measurement equipment.

A wish, as expressed by industrial actors and researchers in the consortium WoodCentre North, is to generate a controlled flow for each individual wood component. This need is for example expressed by saw millers wanting to move away from bulk production to a more dynamic customer-ordered production process in order to utilize the biodiversity of wood better.

For the past decades, the trend has been to group similar logs into bins and sawing batches in the same way (Uusijärvi, 2000). However, in order for the wood industry to take the next step, there is a need to connect the information through the whole chain and adjust processing parameters by means of customer demands and optimizing for value.

Each $\log$ can be divided into several different products. Therefore, a high precision control is needed. The value added to each product, through different processing steps, will be lost if it ends up in the wrong bin. However, high precision control contradicts with keeping a low amount of equipment. It is much more effective to use in-process sensors as monitoring tools than adding new ones which are likely to not get the full service and care by the staff (Flodin, 2009).

A great advantage has emerged due to the possibility to look into the interior of logs with high precision at industrial speeds. The breakdown process can now be carried out virtually and a fingerprint extracted to later be recognized by a surface scanner.

Today, there are image sensors available in the process chain for sorting of logs by quality: 3D shape, discrete X-ray and computed tomography (CT). Surface scanners are present within green and dry sorting of the lumber and a number of process feedback sensors that can be utilized to track and control each individual product in the process. The sensors have different outputs and show different properties or mechanisms depending on sensor type. The problem which remains to be solved is to find appropriate fingerprint parameters and matching algorithms such that the wood products can be recognized at every point in the process.

Can this be done using sensors already present in the chain today, e.g., surface scanners? This article deals with the final stages of a wood value chain, i.e., so called tracking or re-identification of boards.

\subsection{Biometrics}

In the field of biometrics there has been an enormous amount of effort put in improving the recognition of hu- mans (Jain and Kumar, 2012). A lot of different recognition techniques are today being researched in the field of biometric identification for security and prevention of identify theft (Komogortsev and Karpov, 2013; Yang et al., 2013; Czajka and Bulwan, 2013). As wood recognition and wood traceability are relatively unexplored areas of research there is a lot to learn from the human biometrics field. There, the most research and effort has up until today been put into fingerprint, face and iris recognition. However, there are also other applications, for example, palm print, vein, handwriting, sound, gait and ear recognition, that provide inspiration and push the biometrics field forward.

Luckily within wood fingerprint recognition we are spared from problems such as identify theft. However, some similar difficulties can still be present, as well as a few problems which are specific for the recognition of sawn wood products. Like humans, wood can age, which can lead to changes in color but also add crookedness, bow and cracks and so on (Sandberg, 2005). Wood products can also be dried, planed, sanded, treated by some reagent or cut into smaller pieces of different shapes during the processes within which we want to track it. In addition, measurements of wood in industrial processes can be subject to dirty, humid and other adverse environmental conditions. Things like sawdust or dirt, but also lighting can cause problems especially if we are using intensity information directly as feature representation.

The image acquisition can also be negatively affected by loss of traction of conveyor belts or improper clamping of the wood pieces in combination with line scan cameras.

Moreover, while there is a common saying that all trees are as unique as humans, there are also bound to be similar ones, that can cause problems. If thin veneer is cut from one log there will be several similar sheets, or "twins". The front and back of a board can also sometimes look very similar, though the sides will in that case be mirror images of each other.

\subsection{Automatic fingerprint identification systems}

Most often fingerprint identification systems, for humans or wood, need to address the following design steps (Jain et al., 1997):

1. Image acquisition

2. Fingerprint representation

3. Feature extraction

4. Matching 
Systems usually use minutiae and their relative positions as fingerprint representation (Yager and Amin, 2004).

To be able to quickly match against very large databases, a great deal of care must be taken when choosing representation. Identification would often have to be made in haste since modern wood factories have very high flow speeds. For instance, modern sawmills run their conveyors at three meters per second.

Although speed is important in our real-time application, and though speed is always there in the back of our heads, it has not been top priority in this work. There are always strategies to speed up the final identification system. A more thorough optimization of the code will be done at a later stage, after proof of concept.

\subsection{Interest points and feature matching}

Interest points are regions in an image that are likely to be recognized in other images of the same scene or object. Typical interest points include, for example, corners, line endings and blobs (Schmid et al., 2000). Corners, which are positions in an image where there is a strong intensity change in at least two directions, are very good objects to track (Moravec, 1980; Shi and Tomasi, 1994; Rosten and Drummond, 2006).

Usually, a representation of the intensity information in a region around the interest point is stored. These feature representations, or descriptors, can later be used for recognition of objects by matching several feature descriptors between two images.

Criteria for good points to match were described by Förstner (1986) as having the following attributes:

1. Distinctness: The points should be distinguishable from their neighborhood, e.g., consist of a pronounced gradient in intensity or color.

2. Invariance: The points should be invariant with respect to expected geometric and radiometric distortions.

3. Stability: The points should be robust to noise.

4. Seldomness: There should not be several similar points in the same image to avoid confusion. (If a point is part of a repetitive pattern, the possibility for a false match is high.)

5. Interpretability: The points should preferably be interpretable, such as an edge, corner or blob.

Edges however, are usually not good interest points; the region information looks similar along the edge and hence does not fulfill the seldomness requirement. Another example are smooth untextured regions, which do not uphold the distinctness requirement.

There exists a lot of different feature detectors and descriptors. Lowe (2004) proposed an approach called Scale-Invariant Feature Transform (SIFT). SIFT detects the dominant gradient orientation of interest points in an image and saves the gradient information around these points. Since the dominant orientations of the features are calculated, the descriptor becomes rotationally invariant. SIFT features can also be matched between different scales since the images are downsampled iteratively while leaving the kernel size unchanged. A fast and robust detector/descriptor, which in many ways is similar to SIFT, is Speeded-Up Robust Features (SURF, (Bay et al., 2008)). SURF has similar performance as SIFT, but the feature extraction and matching is much faster and the descriptors only allocate half as much storage space by default. Gradient Location-Orientation Histogram (GLOH) is an extension of the SIFT descriptor designed to increase its robustness and distinctiveness. SIFT descriptors are computed in a certain pattern around the interest points and the dimensionality of this descriptor is reduced to 64 by principal component analysis (PCA).

Some of the most popular general-purpose feature descriptors are given in Table 1. Each of them have their

Table 1: A selection of the most popular general-purpose feature descriptors.

\begin{tabular}{ll}
\hline Reference & Descriptor \\
\hline \hline Alahi et al. (2012) & FREAK \\
Bay et al. (2008) & SURF \\
Calonder et al. (2010) & BRIEF \\
Leutenegger et al. (2011) & BRISK \\
Lowe (2004) & SIFT \\
Mikolajczyk and Schmid (2005) & GLOH \\
Rublee et al. (2011) & ORB \\
Tola et al. (2010) & DAISY
\end{tabular}

own strengths and weaknesses, and it is difficult to know beforehand which detector/descriptor combination will perform best for a certain dataset. Extensive evaluations of different detectors and descriptors have been carried out by Mikolajczyk and Schmid (2005) and Gauglitz et al. (2011). We decided to use the two combinations of detectors and descriptors implemented in MATLAB R2011b since they are considered to be state-of-the-art methods and have been widely used in various tracking and object recognition systems (Gauglitz et al., 2011). 


\section{Material}

To test a wood fingerprint recognition method, a large database and preferably also quite many query images, are needed. However, large image sets of boards that have been scanned twice at moderately different settings, are not very common. The two versions of the images should also not be too similar and perfect, as this would render the problem quite trivial. Thus, the decision was made to re-scan a few available floorboards in lab environment.

In the near future, data will be collected from the scanner stations at the Hol-i-Wood Patching Robot plant.

\subsection{Original dataset (database images)}

The chosen dataset consists of 886 floorboard images from Scots pine (Pinus sylvestris L.) with the dimension $21 \times 137 \mathrm{~mm}$ (Figure 1). The same dataset was previously used by Pahlberg and Hagman (2012) and the boards were originally scanned prior to a customer preference study in 2006 (Nyström et al., 2008; Broman et al., 2008; Oja et al., 2008).

The boards are between 3007-5109 mm long, with an average length of approximately $4500 \mathrm{~mm}$. They were sawn from 222 logs with top diameter between 201-215 $\mathrm{mm}$ and were randomly collected from Bollsta sawmill in central Sweden. The boards were then planed, sanded and finished with white pigmented oil and a thin layer of varnish. The finished floorboards were scanned using a high resolution color line scan camera; Dalsa Trillium TR-37. It is a three-CCD camera with a 2048 pixel array for each of the colors; red, green and blue (Nyström et al., 2008). The boards were scanned at a resolution of 2.5 pixels $/ \mathrm{mm}$ lengthwise and 10 pixels $/ \mathrm{mm}$ across. Photocells were set up to automatically crop off $80 \mathrm{~mm}$ from each side to avoid edge artifacts. The images were then downsampled, using the function imresize in MAT$\mathrm{LAB}$, to a resolution of $1 \mathrm{pixel} / \mathrm{mm}$ in both dimensions using the bicubic interpolation setting and the default low-pass filter to mitigate aliasing. (Aliasing may appear as stair-step patterns at lines in an image or as Moiré patterns, a ripple- or wave-type artifact.)

Colors in the images were carefully calibrated to match the colors of the boards in reality. Then the images were saved in JPEG format at $95 \%$ quality using MATLAB's imwrite function.

\subsection{Re-scanned dataset}

The same line scan camera as before was used by Pahlberg and Hagman (2012) to re-scan 44/886 $\approx 5 \%$

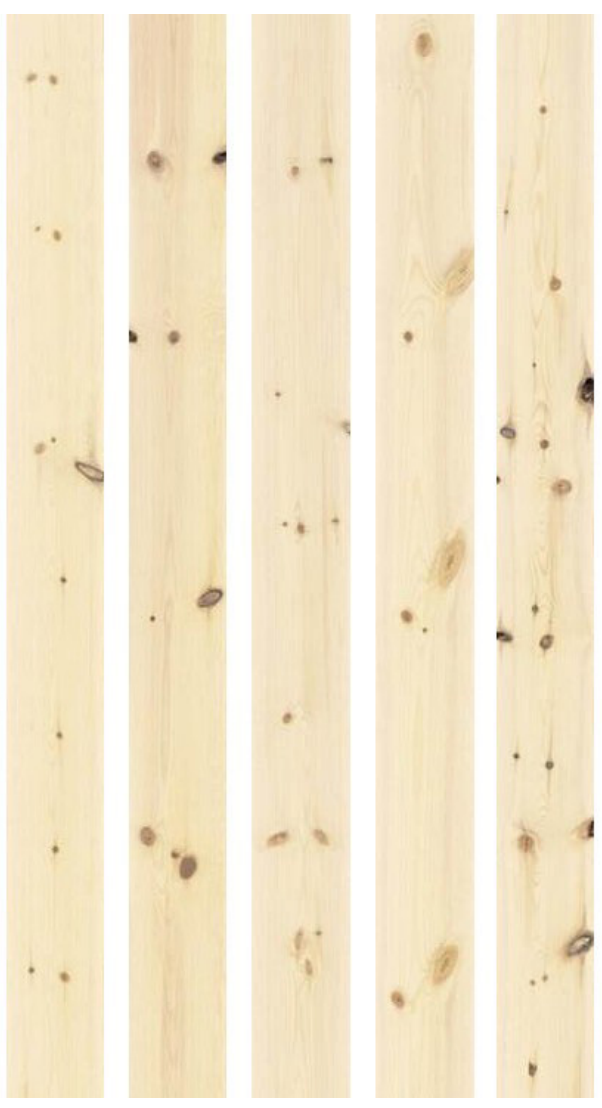

Figure 1: Example of five boards from the original floorboard image dataset. The boards have been planed, sanded and finished with white pigmented oil. Their lengths have been cropped to $734 \mathrm{~mm}$ in this figure.

of the floorboards to have a subset of realistically acquired images to test the matching capabilities on. The boards were randomly picked without considerations to which log they originated from because of convenience. The picked boards have on average $29 \pm 5$ knots ( $\mathrm{min}=$ 18 , $\max =42$ ) of different sizes and appearances. Some of these boards had during the past years of storage become somewhat crooked or bowed. Moreover, no stabilization of the boards was carried out this time while the conveyor belt was transporting them past the camera. No color corrections were made on the images and some dust stains were present on a few boards.

This time, the camera was mounted at such a distance from the conveyor belt that yielded a resolution of 5.6 pixels $/ \mathrm{mm}$ lengthwise and 6.5 pixels $/ \mathrm{mm}$ across. The entire lengths of the board images were kept this time, and the images were downsampled to $1 \mathrm{pixel} / \mathrm{mm}$ using the same method as before for consistency. They were saved in JPEG format as before but now at maximum quality. 


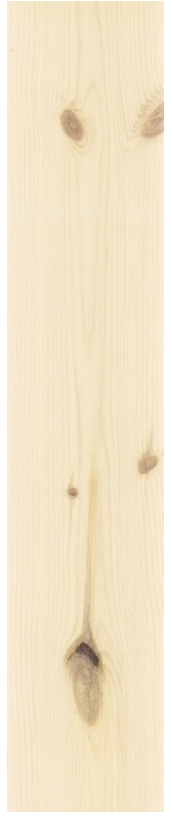

(a) Original.

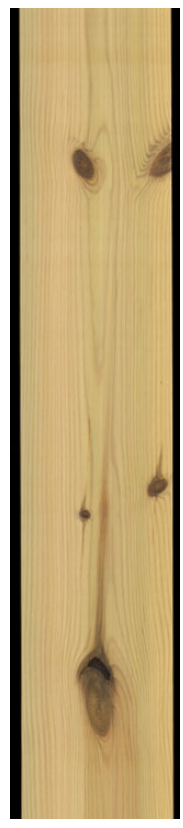

(b) Re-scan.

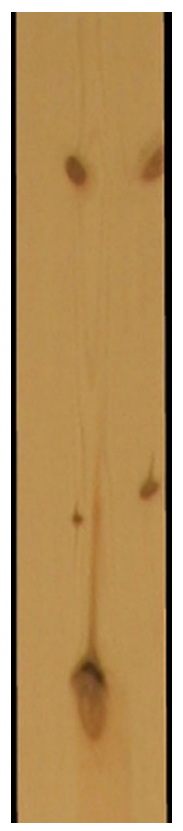

(c) Canon.
Figure 2: Examples of the cropped datasets with an approximate number of knots per image. Figures (a)-(c) are part of the 5-knot batch. Cropped versions of the original images are shown here as reference.

\subsection{Digital photos dataset (Canon)}

Lower quality digital photos were taken of the same 44 floorboards from a distance to provide a tougher challenge for the recognition methods. The photos were acquired with a Canon $400 \mathrm{D}$ camera at a resolution of 0.6 pixels $/ \mathrm{mm}$. The images were upsampled to $1 \mathrm{pixel} / \mathrm{mm}$ in order not to disadvantage the scaledependent block matching method. Scaling was again carried out using MATLAB's imresize and the bicubic interpolation setting.

\subsection{Cropped image datasets}

Several subgroups of images were created from the 44 high- and low quality images. These images were cropped to shorter lengths so that each subgroup would contain board pieces with a specific number of knots, from $5,6, \ldots, 30$ knots. The goal was to show how the different methods' performances vary with increased number of knots. Figure 2 shows examples of a wood piece from the group with five knots. A cropped version of the original dataset image is shown here as a reference, but when matching, the whole length of the original images were always used.

The amount of images in each group can be seen in Figure 3. Naturally, it was not possible to extract as

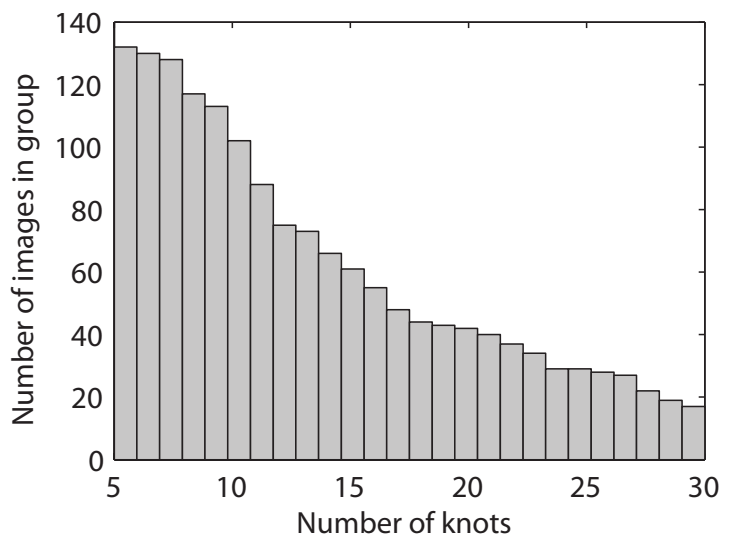

Figure 3: The amount of images in each group of the cropped datasets.

many cropped images with a large amount of knots as with few knots, since in the latter case, many pieces could be grabbed from each board.

All knot positions (pixel coordinates) were marked manually in the computer to be able to determine good possible crop positions. A script was written to automatically cut the images in between knots. However, sometimes for Scots pine there are two or three knots at the same lengthwise position. This circumstance led to a few knots being cut in half. Nonetheless, the average number of knots in the images of each batch should be close to the target number.

Very small knots were counted even though they would probably not generate any stable interest points. However, very small knots on edges of the boards were not counted since features on edges of boards are removed in the matching algorithm. Some marginal differences in the crop positions between the high- and low quality dataset can occur, since the very smallest knots were not always visible in the low quality dataset, and were therefore not marked.

An overview of all different datasets can be found in Figure 4.

\section{Methods}

In this section the feature detection algorithms and the matching procedures are explained in order to be able to replicate the approach.

\subsection{Feature detection and matching}

Boards can be identified by matching many small features between image pairs. The feature descriptors of a query board are compared against the feature descriptors of all the boards in a database. The database board 


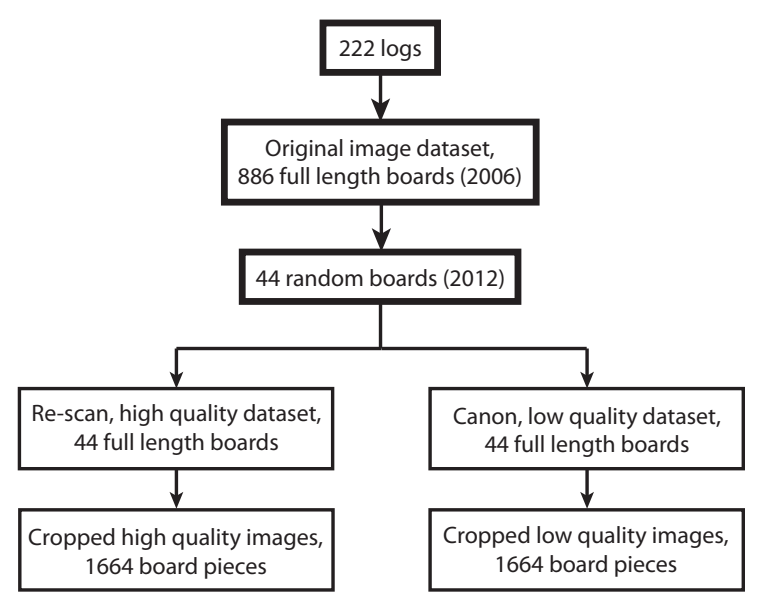

Figure 4: An overview of the different datasets used in this paper. The 44 boards were randomly picked without considerations to which log they originated from because of convenience.

with highest number of matching feature descriptors is deemed to be the correct match.

Two different feature detection methods have been utilized individually, but also in combination, to carry out the identification of boards in the experiments. The first method will be referred to as the Block matching method (Nistér et al., 2004; MathWorks MATLAB; Szeliski, 2011, p. 222). The second method will be referred to as the SURF matching method (SpeededUp Robust Features, (Bay et al., 2008)), and finally, the combination of the two will be denoted the Fused method. An example that illustrates how the feature descriptors are being placed on the different datasets can be seen in Figure 5.

The procedure for finding a best matching wood piece in the database is similar for the three methods and can roughly be summarized as follows:

1. Load a query image.

2. Invoke a corner/blob detector on the image and extract feature descriptors around those corners/blobs, (MATLAB functions: detectHarrisFeatures, detectSURFFeatures, extractFeatures).

3. Remove descriptors that are partly outside of the wood piece border by checking if they include intensity values equal to zero.

4. Match query descriptors against database descriptors, one database image at a time, (MATLAB function: matchFeatures).

5. Estimate a best fitting geometric transformation between point matches and remove outlier matches

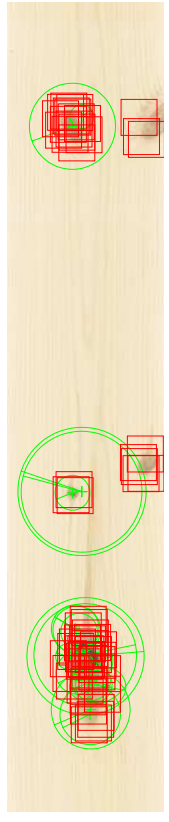

(a) Original.

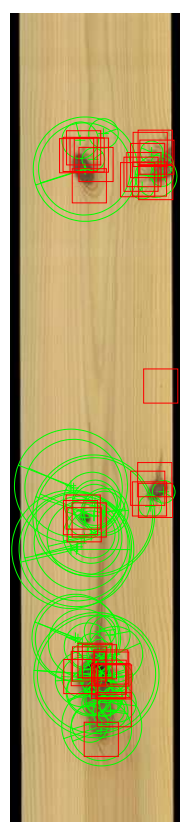

(b) Re-scan.

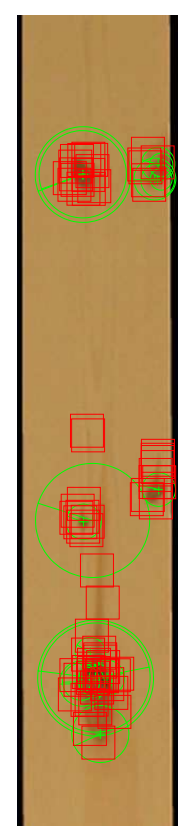

(c) Canon.
Figure 5: Examples of how the different feature descriptors are being placed on the different datasets (same images as in Figure 2). Block features are shown with red squares and SURF features with green circles.

that don't fit the transformation, (MATLAB function: estimateGeometricTransform).

6. Evaluate if the transformation seems reasonable in terms of rotation and scaling. Reject the matched database board if the rotation or scaling is too large.

7. Remove clusters of $\geq 4$ feature points within a $3 \mathrm{~cm}$ radius, (MATLAB functions: ExhaustiveSearcher, rangesearch).

The database image that has the most matching features is denoted the best match of the query. If two or more database images get the same amount of matching features, no unique match was found and thus the wood piece could not be identified.

Two experiments were created to be able to answer the research questions, i.e., (i) to find out if it is at all possible to correctly re-identify wooden boards and (ii) to find out exactly how small wood pieces can be recognized.

(i) Match the full length re-scanned images and Canon images against the original dataset.

(ii) Match the cropped images in each group against the full length original images. 
The matching accuracy is used to evaluate the results and is defined as:

\section{Number of correct identifications}

$$
\text { Number of images in dataset, or group }
$$$$
100(\%)
$$

In this paper we only match images unrotated, or having very small rotation angles that were induced when scanning. The rotationally dependent Block matching method has been proven to work well for small rotations (Pahlberg and Hagman, 2012), but it cannot handle, for example, 180 degree rotations as it is now. Such added functionality would not be very difficult to implement but would increase the computation time.

Many components of the feature detection methods used in this paper can be found as open source implementations (Bradski, 2000), and they are often used for similar purposes in other computer vision applications.

In this paper, implementations from MATLAB ${ }^{\circledR}$ R2013a, Computer Vision System Toolbox ${ }^{\mathrm{TM}}$ have been incorporated.

\subsection{Block matching method}

In the Block matching method the normalized intensity information in simple square regions are extracted as feature descriptors around corners in an image (Figure 6(a)). Descriptors were extracted using the MATLAB-function extractFeatures. The block size was set to $25 \times 25$ pixels, as a compromise between a large enough size to encircle substantial parts of the knots and a not too large size as to incur slow computation times. Relatively small regions also give us the possibility to extract features close to wood piece edges, as features lying directly on an edge are rejected. A detailed explanation of the procedure is given in Algorithm 1.

Interest points were obtained using Harris corner detector (Harris and Stephens, 1988) with MATLAB's function detectHarrisFeatures, as opposed to our previous work (Pahlberg and Hagman, 2012), where the FAST corner detector was used (Rosten and Drummond, 2006). The FAST corner detector was found to return too much false corners along jagged lines; hence a switch of corner detector was made. A Gaussian filter of size $5 \times 5$ pixels and $\sigma=5 / 3$ is used as part of the Harris corner detector to smooth gradients and reduce noise before the actual corner extraction in all dataset images. The threshold for accepting corners is set as a fraction of the maximum corner metric value in that image. This fraction was lowered to 0.005 instead of the default value 0.01 in order to retrieve more corners. However, no limit was set on the amount of corners to be returned.

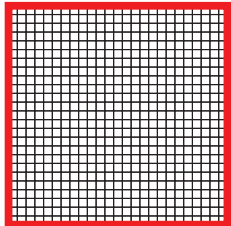

(a)

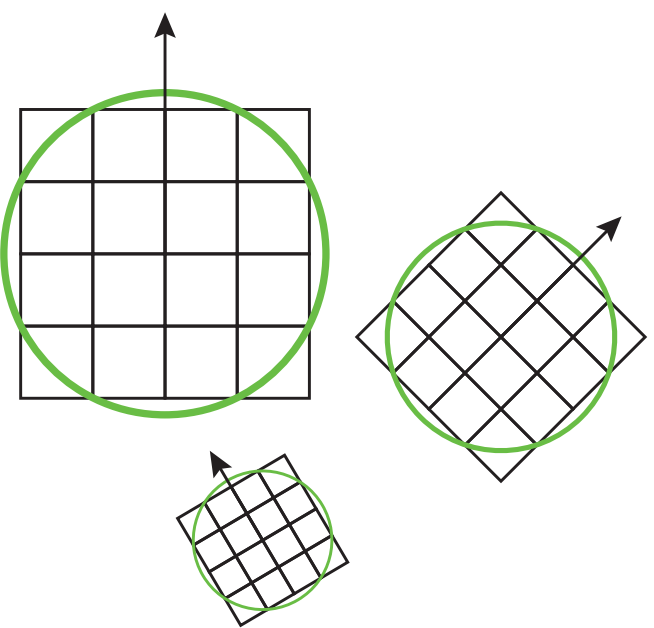

(b)

Figure 6: (a) A simple $25 \times 25$ Block feature. (b) Three SURF features of different sizes and orientations. The interest points' dominant directions are marked by the arrows.

The MATLAB-function matchFeatures carries out the matching procedure. The function takes two sets of features, matches these and returns a list of indices connecting the features that match. For comparing blocks, normalized Sum of Squared Differences (SSD) was chosen as metric. A threshold for rejecting matches is specified as a percentage of the worst case scenario for the SSD between two normalized vectors. This percentage is then converted to a numeric value between the best case and the worst case; on the interval $[0,4]$. The threshold for rejecting matching blocks was increased from $1 \%$ to $2 \%(\Rightarrow 0.02 \cdot 4)$ to approve more matches than the default.

\subsection{SURF matching method}

Speeded-up Robust Features (SURF) are robust scale- and rotationally invariant features which are fast to compute and suitable for object detection and recognition. This feature detector was chosen because of its popularity and since it was readily available.

The features are made invariant to rotation by aligning them along the dominant direction of the interest point within a circular neighborhood (Figure 6(b)). 
(However, in this study, the scale- and rotational invariance does not come into play, since all images are of roughly of the same scale and rotation.)

SURF uses integral images (Viola and Jones, 2001) for fast calculations of sums of intensities over square regions in grayscale images. The use of integral images makes it possible to do such computations in constant time, independent of region size. The scale space (image pyramid) is created by iteratively up-scaling kernel sizes instead of iteratively downsampling the image, which is more computationally expensive. The SURF descriptor is a $16 \times 4=64 \mathrm{D}$-vector, representing normalized gradient statistics (mean and absolute mean values) extracted from a spatial grid divided into 4-by-4 cells (Oyallon and Rabin, 2013).

SURF is thoroughly described in Bay et al. (2008), and an extensive algorithm description can be found in Oyallon and Rabin (2013). A condensed version is presented here in Algorithm 2.

The SURF features are detected, extracted and matched using MATLAB-functions detectSURFFeatures, extractFeatures and matchFeatures. Some key settings used for the SURF method in our implementation is given in Table 2. These settings will extract descriptors of sizes between $9 \times 9$ and up to $147 \times 147$ pixels (or $\mathrm{mm}$ ) in the images. The threshold for accepting interest points was lowered substantially compared to our previous work (Pahlberg and Hagman, 2012), where the default value was used.

The SSD percentage threshold is also here converted to a numerical value $(5 \% \Rightarrow 0.05 \cdot 4)$ in matchFeatures.

Table 2: Settings for SURF in our implementation

\begin{tabular}{lrr}
\hline Parameter & Our value & Default \\
\hline \hline Scale-space octaves & 3 & 3 \\
Scale levels within octaves & 6 & 4 \\
Thresh. to accept features & 150 & 1000 \\
Max no. of SURF features & $\infty$ & $\infty$ \\
SSD thresh. to accept match & $5 \%$ & $1 \%$ \\
\hline
\end{tabular}

\subsection{Removing false matches}

Some features on wood, such as knots, can look quite similar, especially to a computer that hasn't been trained properly or using enough parameters. Knots are usually somewhat circular and similar in size and color. Often there will be a few very similar looking knots on a board. This ambiguity leads to the possibility that a feature in one image can be matched to several

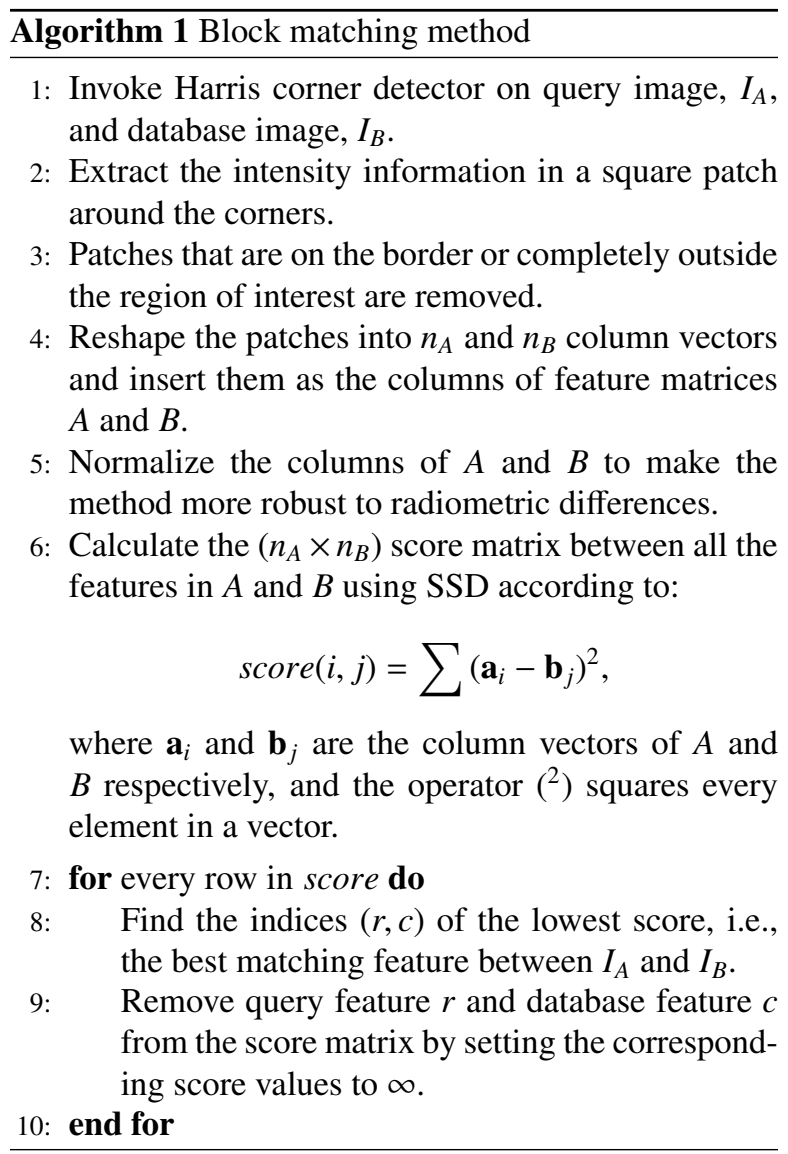

features in another image. To take care of this problem, the method NearestNeighborSymmetric was chosen in MATLAB for matching both Block- and SURFfeatures. This method only returns unique matches in addition to using the match threshold. A feature vector is matched to its nearest neighbor in the other feature set, and for a match to be accepted, both points must be the best matching feature of the other (side symmetry).

MATLAB's Geometric Transform Estimator (GTE) was utilized to estimate a fitting geometric transformation between the locations of the interest points and to exclude outlier matches. The computationally simple non-reflective similarity transformation was chosen since we are dealing with small and simple geometric distortions. The similarity transformation consists of an isotropic scaling, a rotation and a translation (Hartley and Zisserman, 2003). This means that the shapes of the objects are preserved, which is a reasonable approximation for an application with wooden boards if the cameras are set up properly.

The Euclidean pixel distance threshold was set to 100 to allow for some deviation from the exact transforma- 


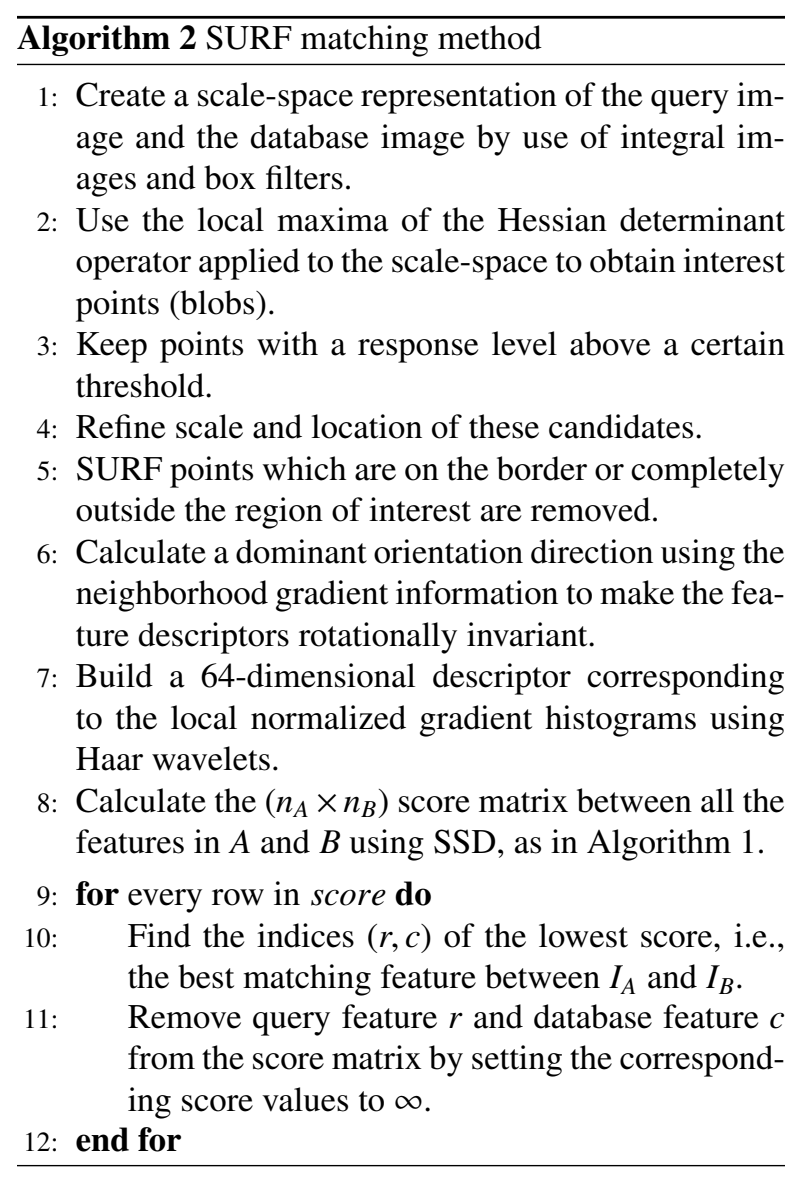

tion, mainly to handle warp of the boards. Random Sample Consensus, (RANSAC, (Fischler and Bolles, 1981)), was chosen as the method for determining inlier matches. The GTE with RANSAC iteratively picks a few matched point pairs, estimates a transformation and checks how many inliers are within a threshold. The transform with most inliers is chosen as the correct one and the inliers are the accepted matches.

A problem may arise where many features in a tight cluster in one image are sometimes matched to scattered points in the other image (Figure 7(a)). This problem was taken care of by investigating the transformation, $T$, returned by the GTE. If the similarity transformation led to a scaling of more than $5 \%$, or if the rotation angle was more than 5 degrees, the database image would be discarded.

If four or more features were found within a $3 \mathrm{~cm}$ radius after the GTE, the cluster of features was discarded altogether as the features were deemed to be too ambiguous, see example in Figure 7(b).

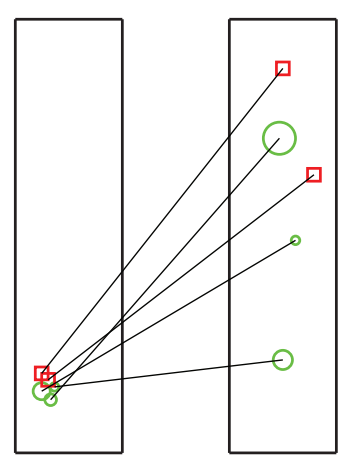

(a)

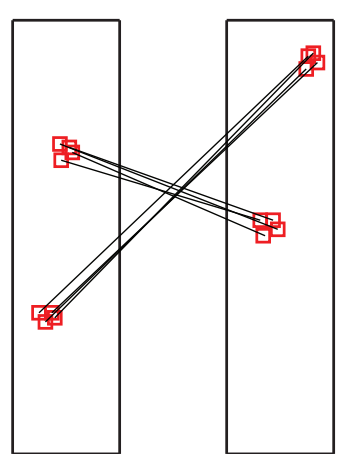

(b)
Figure 7: Two of the most common types of false matches. (a) A tight cluster of features falsely matched to sparsely scattered features. (b) Highly ambiguous features that are falsely matched. (Block features are marked by red squares and SURF features with green circles.)

Table 3: Matching accuracy in \% using entire board images as queries.

\begin{tabular}{lrrr}
\hline Dataset & Block & SURF & Fused \\
\hline \hline Re-scanned & 93.1 & 97.7 & 100.0 \\
Canon & 93.0 & 81.4 & 97.7 \\
\hline
\end{tabular}

\section{Results}

\subsection{Matching full length boards}

When entire board images are used as queries, all 44 boards are found using the Fused method for the high resolution dataset (Table 3). The Block matching method has similar performance for both datasets in this test. The SURF method performs somewhat better on the higher resolution dataset.

Matching of whole boards was also done in our previous work (Pahlberg and Hagman, 2012). Compared to the previous results, the switch to Harris corner detector for the Block matching method dramatically increased the matching accuracy on the low resolution Canon dataset, from $9.1 \%$ to $93.0 \%$. For the high resolution dataset, the accuracy decreased slightly, from $95.5 \%$ to $93.1 \%$. The SURF method increased the recognition accuracy from $34.1 \%$ to $81.4 \%$ on the low quality dataset. For the high quality dataset, the accuracy was increased from $86.4 \%$ to $97.7 \%$.

Through screening tests it was found that the thresholds for accepting interest points affected the matching accuracy the most. In Table 4, the impact of the new threshold choices can be seen compared to the default values, and what impact this change has on inliers, execution time and matching accuracy. The matching accuracy is most positively affected when using the low 
quality dataset. Notable is also that the matching accuracy is high even when the inlier percentage is quite low. This fact highlights the importance of the geometric transform estimator with RANSAC.

\subsection{Matching cropped images}

An example of two successfully matched cropped images can be seen in Figure 8(a)-(b), for the high quality and the low quality dataset respectively. Both methods often find a good amount of matching features when using the high resolution images. However, when using the low resolution images, the SURF method usually finds fewer features, see Figure 8(b).

In Figure 9(a)-(b) the matching accuracy is plotted against the number of knots of each group. The accuracy increases quite rapidly with increasing number of knots for all methods and both datasets. For all methods the increase in matching accuracy starts to slow down between 10-15 knots.

The fusion of the two methods performs best and reaches $100 \%$ matching accuracy for board pieces with more than 20 knots for the high resolution dataset. For the low resolution dataset, the matching accuracy is close to $100 \%$ after 20 knots but seemingly drops somewhat when boards contain more knots. This drop was caused by one particular board that could not be found, where the majority of the features were falsely matched. The slope of the curve is negative here since the last knot groups contain fewer boards.

The SURF matching method performs better on the high resolution dataset than on the low resolution counterpart for all numbers of knots. The SURF method and the Block method have similar performance on the high resolution dataset independent of the amount of knots.

Both methods usually extract more than double the amount of features from the high resolution dataset compared to the low resolution.

Noteworthy is that the simple Block matching method has a substantially higher performance on the lower resolution digital photos compared to the SURF matching method. The Block method also performs better on the low resolution images than on the higher resolution images between 5-15 knots. Between 15-30 knots, there is no distinguishable performance difference between the datasets for the Block method.

\section{Discussion}

There are a lot of parameters that can be tweaked to calibrate and improve the performance of identification systems that utilize feature detectors. The sensitivity of

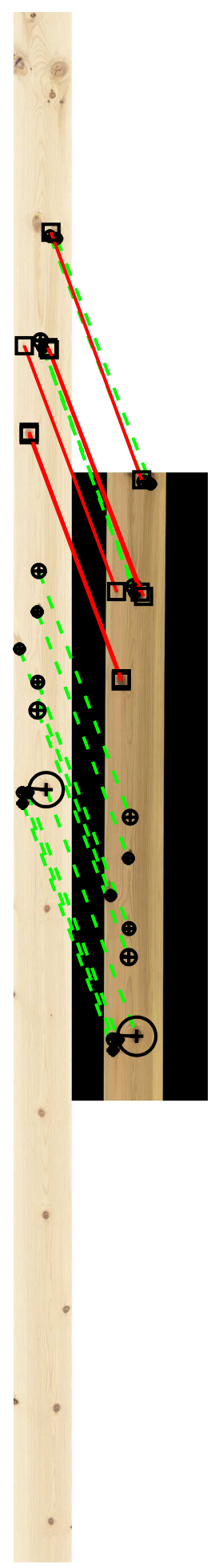

(a)

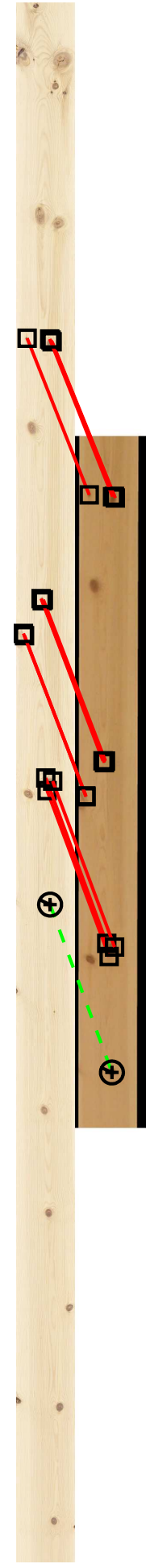

(b)
Figure 8: Two successful matchings from the cropped image group with eight knots using the Fused method. (a) Re-scanned dataset. (b) Canon dataset. The Block features are marked by squares and matches are connected by solid red lines. SURF features are marked by circles 
Table 4: Matching statistics for the 44 full-length boards while changing corner/blob threshold. A mean has been calculated for \# inliers, \# interest points, $\%$ inliers and execution time. The statistics are given on the format: mean (standard deviation,min, max). All values except matching accuracy have been calculated using only the observations in which the methods found the correct database board. \% inliers is calculated as mean (\# inliers / \# interest points) $\times 100$. Matching accuracy is calculated as in Equation (1).

(a) Block matching statistics (Re-scan dataset)

\begin{tabular}{lrrrrrr}
\hline Corner threshold & Obs & \# inliers & \# interest points & \% inliers & Exec. time (s) & Acc. $(\%)$ \\
\hline \hline 0.01 (default) & 40 & $18(8,5,35)$ & $88(51,15,219)$ & $26(12,7,55)$ & $18(4,11,25)$ & $\mathbf{9 1}$ \\
0.005 (new) & 41 & $22(8,9,41)$ & $147(68,29,299)$ & $19(10,5,52)$ & $27(8,13,41)$ & $\mathbf{9 3}$ \\
\hline
\end{tabular}

(b) Block matching statistics (Canon dataset)

\begin{tabular}{lrrrrrr}
\hline Corner threshold & Obs & \# inliers & \# interest points & \% inliers & Exec. time (s) & Acc. (\%) \\
\hline \hline 0.01 (default) & 36 & $14(5,5,26)$ & $48(27,8,107)$ & $36(16,9,70)$ & $13(3,9,20)$ & $\mathbf{8 4}$ \\
0.005 (new) & 40 & $18(7,5,36)$ & $77(42,17,193)$ & $29(13,7,65)$ & $18(5,11,28)$ & $\mathbf{9 3}$ \\
\hline
\end{tabular}

(c) SURF method statistics (Re-scan dataset)

\begin{tabular}{lrrcccr}
\hline Blob threshold & Obs & \# inliers & \# interest points & \% inliers & Exec. time (s) & Acc. $(\%)$ \\
\hline \hline 1000 (default) & 41 & $11(4,5,22)$ & $44(10,23,69)$ & $24(8,13,44)$ & $10(0,9,11)$ & $\mathbf{9 3}$ \\
150 (new) & 43 & $17(7,6,39)$ & $183(62,81,329)$ & $10(4,4,19)$ & $16(2,13,21)$ & $\mathbf{9 8}$ \\
\hline
\end{tabular}

(d) SURF method statistics (Canon dataset)

\begin{tabular}{lrrccrr}
\hline Blob threshold & Obs & \# inliers & \# interest points & \% inliers & Exec. time (s) & Acc. $(\%)$ \\
\hline \hline 1000 (default) & 29 & $7(2,5,14)$ & $30(8,14,44)$ & $25(9,12,50)$ & $10(1,9,11)$ & $\mathbf{6 7}$ \\
150 (new) & 35 & $8(3,4,15)$ & $50(13,28,85)$ & $17(6,8,34)$ & $11(1,9,12)$ & $\mathbf{8 1}$ \\
\hline
\end{tabular}

the interest point detectors and the thresholds to accept point matches are the most obvious. One improvement would be to implement a smarter approach for accepting interest points. In the Block matching method, the Harris corner acceptance threshold is adaptive and depends on the highest corner response value in that image. This is not appropriate if one corner value is much higher than the others. It can lead to less distinct knots not being found. Another solution would be to detect a certain number of corners, for example, the 300 strongest corners. This solution would also make it possible to have control over the maximum computation time. The optimal window size for the corner/blob detector, together with the appropriate amount of a priori smoothing of the image, must also be investigated.

The fusion of the two methods performs best, which was expected. Therefore, we recommend a combination of different feature detectors as they complement each other and react to different positions in an image. More extracted features in general seem to increase the chances of finding the right wood piece but at the expense of computation time.

As can be seen in Figure 8(b), the SURF method had only one matching feature in this particular case. A solution could be to lower the threshold for accepting blobs, or to change the algorithm to allow feature descriptors to have more than one match each.

The simple Block matching method showed a surprisingly high performance for the lower resolution digital photos. This implies that a priori blurring of the query images could possibly have a positive effect on the performance of the Block method. The reason why the SURF method performs worse on this dataset is probably because its blob detector does not extract as many, or as well spread out, interest points as the Harris corner detector. Often Harris corner detector finds more interest points around each knot compared to the SURF method's determinant of Hessian blob detector which often only finds 1-2 points on the low resolution images. The SURF method's blob detector extracts more interest points on the high resolution dataset, where it reacts to places seemingly even on clearwood. The scale- and rotational invariance of SURF should not yield any special advantage nor disadvantage in our tests, since both query and database images were approximately unrotated and of the same scale.

The quality of the input images certainly has an impact on the recognition accuracy. The smoothness of 


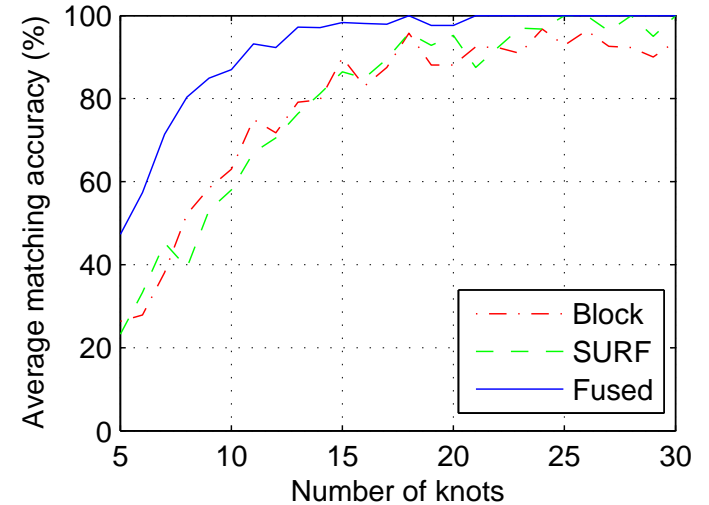

(a)

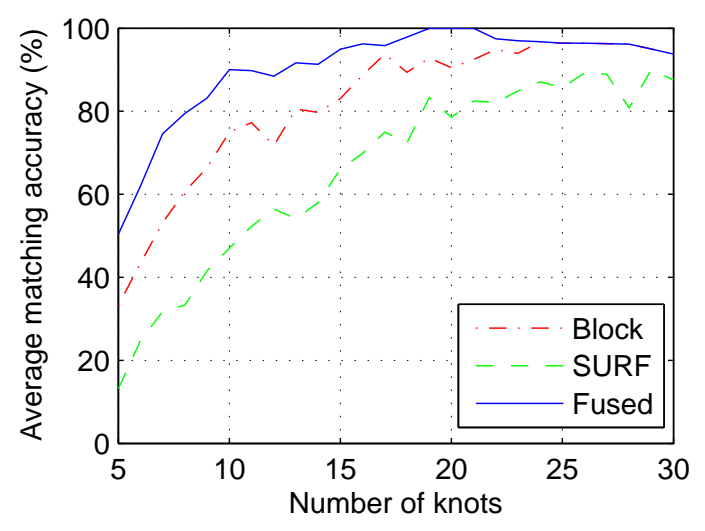

(b)

Figure 9: (a) Matching accuracy of subsets of the Re-scanned floorboards. (b) Matching accuracy of subsets of the digital photos.

the board surfaces would also affect the result. However, preliminary tests have shown that rough sawn surfaces yield more interest points than smooth surfaces like on our floorboards. This fact should translate to better recognition possibilities for rough sawn products. The staining with white pigmented oil used in this study should not give any special advantage nor disadvantage since the feature descriptors normalize the intensity within each feature descriptor region.

In this work, all boards in the database were always traversed in a "brute-force" manor. To increase speed, the search space could be reduced by grouping similar wood fingerprints into bins or by sorting similar boards after some measure. Measures such as for example the variance of colors or the average distance between knots, could be used. Moreover, parallelization could be implemented, utilizing multi-core processors or graphic cards, as most of the computations are independent of each other.

\section{Conclusions}

- The fusion of the Block matching method and the SURF method improved the recognition rate of wooden boards substantially over the individual feature detection methods.

- $100 \%$ matching accuracy was obtained on our Scots pine board pieces with more than 20 knots using high quality images. Low quality images yield results close to $100 \%$ for the same case.

- More than $90 \%$ matching accuracy was achieved for board pieces with more than 10 knots, using both high- and low quality images.

- The individual feature detection methods extract much more features from high quality images than from those of low quality.

- The recognition rate could be increased for boards with few knots by extracting more interest points, but at the expense of longer computation time.

\section{Future work}

Future work will include investigation of how knot positions and other geometric relationships between knots could be utilized for recognition. Moreover, there is a need to further investigate which corner/blob detectors and feature descriptors are the most suitable for different kinds of wood surfaces and given different types of noise.

\section{Acknowledgements}

This work has been part of the Hol-i-Wood Patching Robot project and received funding from the European Union's Seventh Framework Programme (FP7/20072013) under grant agreement no. 284573. The Hol-iWood PR is a collaboration between Luleå University of Technology (LTU), TU Wien and TU München, as well as industrial partners; MiCROTEC, Springer, TTTech and LIP-BLED.

\section{References}

Alahi, A., Ortiz, R., Vandergheynst, P., 2012. FREAK: Fast Retina Keypoint, in: IEEE Conference on Computer Vision and Pattern Recognition, Ieee, New York. CVPR 2012 Open Source Award Winner.

Bay, H., Ess, A., Tuytelaars, T., Van Gool, L., 2008. Speeded-Up Robust Features (SURF). Computer Vision and Image Understanding $110,346-359$. 
Björk, A., Erlandsson, M., Häkli, J., Jaakkola, K., Nilsson, Å., Nummila, K., Puntanen, V., Sirkka, A., 2011. Monitoring environmental performance of the forestry supply chain using RFID. Computers in Industry $62,830-841$.

Bradski, G., 2000. The OpenCV Library. Dr. Dobb's Journal of Software Tools

Broman, N.O., Nyström, J., Oja, J., 2008. Modelling the connection between industrially measured raw material properties and end user preferences. Part 2 - Results from preference studies, in: Proceedings of the IUFRO Working Party 5.01.04, Joensuu, Finland.

Calonder, M., Lepetit, V., Strecha, C., Fua, P., 2010. Brief: Binary robust independent elementary features, in: Proceedings of the 11th European Conference on Computer Vision: Part IV, SpringerVerlag, Berlin, Heidelberg. pp. 778-792.

Chiorescu, S., Grönlund, A., 2004. The fingerprint method: Using over-bark and under-bark log measurement data generated by three-dimensional log scanners in combination with radiofrequency identification tags to achieve traceability in the log yard at the sawmill. Scandinavian Journal of Forest Research 19, 374383.

Czajka, A., Bulwan, P., 2013. Biometric verification based on hand thermal images, in: Biometrics (ICB), 2013 International Conference on, pp. 1-6.

Deng, H., Zhang, W., Mortensen, E., Dietterich, T., Shapiro, L., 2007. Principal Curvature-Based region detector for object recognition, in: 2007 IEEE Conference on Computer Vision and Pattern Recognition, IEEE. pp. 1-8.

Dykstra, D.P., Kuru, G., Taylor, R., Nussbaum, R., Magrath, W.B., Story, J., 2002. Technologies for Wood Tracking, In: Environmental and Social Development East Asia and Pacific Region Discussion Paper. Technical Report. World Bank.

Fischler, M.A., Bolles, R.C., 1981. Random sample consensus: A paradigm for model fitting with applications to image analysis and automated cartography. Comm ACM 24, 381-395.

Flodin, J., 2009. KAP - Kontinuerlig Automatisk Provsågning. Technical Report. WoodCenter North.

Flodin, J., Oja, J., Grönlund, A., 2008. Fingerprint traceability of sawn products using industrial measurement systems for x-ray log scanning and sawn timber surface scanning. Forest Products Journal $58,100-105$.

Förstner, W., 1986. A feature based correspondence algorithm for image matching. International Archives of Photogrammetry, Remote Sensing, and Spatial Information Sciences 26, 150-166. Rovaniemi.

Gauglitz, S., Höllerer, T., Turk, M., 2011. Evaluation of interest point detectors and feature descriptors for visual tracking. Int. J. Comput. Vision 94, 335-360.

Grönlund, A., 2008. SkeWood - Ett forskningsprogram vid Luleå tekniska universitet. Technical Report 25. LTU Skellefteå.

Häkli, J., Sirkka, A., Jaakkola, K., Puntanen, V., Nummila, K., 2013. Challenges and Possibilities of RFID in the Forest Industry, Radio Frequency Identification from System to Applications, Dr. M. I. B. Reaz (Ed.). InTech. chapter 15. pp. 301-324.

Harris, C., Stephens, M., 1988. A Combined Corner and Edge Detection, in: Proceedings of The Fourth Alvey Vision Conference, pp. 147-151.

Hartley, R.I., Zisserman, A., 2003. Multiple View Geometry in Computer Vision. 2nd ed., Cambridge University Press, ISBN: 0521540518.

Jain, A., Hong, L., Pankanti, S., Bolle, R., 1997. An identityauthentication system using fingerprints. Proceedings of the IEEE $85,1365-1388$

Jain, A.K., Kumar, A., 2012. Biometric recognition: An overview, in: Mordini, E., Tzovaras, D. (Eds.), Second Generation Biometrics: The Ethical, Legal and Social Context. Springer Netherlands. volume 11 of The International Library of Ethics, Law and Technology, pp. 49-79.

Komogortsev, O.V., Karpov, A., 2013. Liveness detection via oculomotor plant characteristics: Attack of mechanical replicas, in: 2013 International Conference on Biometrics (ICB), pp. 1-8.

Leutenegger, S., Chli, M., Siegwart, R.Y., 2011. Brisk: Binary robust invariant scalable keypoints, in: Proceedings of the 2011 International Conference on Computer Vision, IEEE Computer Society, Washington, DC, USA. pp. 2548-2555.

Lowe, D.G., 2004. Distinctive image features from scale-invariant keypoints. International Journal of Computer Vision 60, 91-110.

MathWorks MATLAB, 2013. Computer Vision System Toolbox: 2013 b - Video Stabilization Using Point Feature Matching. The MathWorks Inc., Natick, Massachusetts, United States.

Mikolajczyk, K., Schmid, C., 2005. A performance evaluation of local descriptors. IEEE Transactions on Pattern Analysis \& Machine Intelligence 27, 1615-1630

Moravec, H., 1980. Obstacle Avoidance and Navigation in the Real World by a Seeing Robot Rover. Technical Report CMU-RI-TR80-03.

Nistér, D., Naroditsky, O., Bergen, J., 2004. Visual odometry, in: IEEE conferenceon computer vision and pattern recognition (CVPR'04), pp. 652-659.

Nyström, J., Oja, J., Broman, N.O., 2008. Modelling the connection between industrially measured raw material properties and end user preferences. Part 1 - The simulation tool, in: Proceedings of the IUFRO Working Party 5.01.04, Joensuu, Finland.

Oja, J., Broman, N.O., Nyström, J., 2008. Modelling the connection between industrially measured raw material properties and end user preferences. Part 3 - Optimizing the industrial production, in: Proceedings of the IUFRO Working Party 5.01.04, Joensuu, Finland.

Oyallon, E., Rabin, J., 2013. An analysis and implementation of the SURF method, and its comparison to SIFT. http://www.ipol.im/pub/pre/69/preprint.pdf, last visited 2014-04-06.

Pahlberg, T., Hagman, O., 2012. Feature recognition and fingerprint sensing for guiding a wood patching robot, in: Quenneville, $\mathrm{P}$. (Ed.), World Conference on Timber Engineering, pp. 724-733.

Põlder, A., Juurma, M., Tamre, M., 2012. Wood products automatic identification based on fingerprint method. Journal of Vibroengineering 14.

Rosten, E., Drummond, T., 2006. Machine learning for high-speed corner detection, in: European Conference on Computer Vision, pp. 430-443.

Rublee, E., Rabaud, V., Konolige, K., Bradski, G.R., 2011. Orb: An efficient alternative to sift or surf., in: Metaxas, D.N., Quan, L., Sanfeliu, A., Gool, L.J.V. (Eds.), ICCV, IEEE. pp. 2564-2571.

Sandberg, D., 2005. Distortion and visible crack formation in green and seasoned timber: influence of annual ring orientation in the cross section. Holz als Roh- und Werkstoff 63, 11-18.

Schmid, C., Mohr, R., Bauckhage, C., 2000. Evaluation of interest point detectors. International Journal of Computer Vision 37, 151172.

Shi, J., Tomasi, C., 1994. Good features to track, in: Computer Vision and Pattern Recognition, 1994. Proceedings CVPR '94., 1994 IEEE Computer Society Conference on, pp. 593-600.

Szeliski, R., 2011. Computer Vision: Algorithms and Applications. Springer London.

Tola, E., Lepetit, V., Fua, P., 2010. DAISY: An Efficient Dense Descriptor Applied to Wide Baseline Stereo. IEEE Transactions on Pattern Analysis and Machine Intelligence 32, 815-830.

Uusijärvi, R., 2000. Automatic tracking of wood. Ph.D. thesis. Kungliga Tekniska Hgskolan. Institutionen för produktionssystem.

Viola, P., Jones, M., 2001. Rapid object detection using a boosted cas- 
cade of simple features, in: Computer Vision and Pattern Recognition, 2001. CVPR 2001. Proceedings of the 2001 IEEE Computer Society Conference on, pp. I-511 - I-518 vol.1.

Yager, N., Amin, A., 2004. Fingerprint classification: a review. Pattern Analysis \& Applications 7, 77-93.

Yang, J., Lei, Z., Liao, S., Li, S.Z., 2013. Face liveness detection with component dependent descriptor, in: 2013 International Conference on Biometrics (ICB), pp. 1-6. 